What difference does it make? Findings of an impact study of service user and carer involvement on social work students' subsequent practice

\begin{abstract}
What difference does it make? Findings of an impact study of service user and carer involvement on social work students' subsequent practice
\end{abstract}

This paper reports on a study which followed up a group of social work students to explore whether service user involvement in their education made any difference to their practice and whether there were different models or types of involvement which had a more significant impact than others. The findings showed that the impact was individual to each student. It was often specific encounters or 'light bulb moments' which led to increased awareness or insight into their own practice particularly when encountering similar situations or when pressures of practice led to them losing sight of service user perspectives. The paper shares these findings and considers the learning for educators in developing models of involvement which enhance impact on subsequent practice. 


\section{What difference does it make? Findings of an impact study of service user and carer involvement on social work students' subsequent practice}

Research into service user involvement in social work education to date has focussed in many cases on evaluating specific activities or models of involvement at the time. Literature resulting from this has shown that students highly evaluate experiences which involve service users and carers; that involvement can motivate students and practitioners to implement changes to their practice in innovative ways (Khoo, McVicar and Brandon, 2004); that involvement improves students' attitudes toward people with particular conditions or experiences (MacSporran, 2015) and can make educators reflect more deeply on the way they communicate (Perry, Gilbert and Rawlinson, 2013). There is evidence that meaningful involvement can facilitate transformative learning (Mezirow, 2001) where students learn to critically question their own beliefs and views of the world and develop a more open and inclusive approach to their practice (Rush, 2008; Author's own, 2012). These are all outcomes which are congruent with the need for social work education to enable students to develop anti-oppressive practice and maintain professional values and standards and to prepare students for the complexities of the workplace where there may not be clear cut solutions.

From our own research at X University, we know that students value service user involvement and report it as being eye opening, humbling and inspiring to hear people's lived experiences and value their contribution to the design and delivery of their programme. They identify how it has enabled them to relate more formal learning to their practice. In an evaluation of a first year BA Social work unit which is service user led (Authors own 2012) for example, one student said "It wasn't until this unit that I really understood the importance of what we had been learning in Uni”. Members of our service user and carer partnership 
report that they feel listened to and feel that they are contributing to the future workforce and making a difference.

There is limited evidence however on what this difference might be. Carr (2004) for example, in a report for the Social Care Institute for Excellence (SCIE) identified that evaluations of service user involvement were more likely to focus on participation techniques, than on successful impact and outcomes. Morgan and Jones' (2009) and Robinson and Webber's (2013) literature reviews (in health education and social work education respectively) both concluded that whilst students and service users valued involvement in their education, there was little evidence in the literature of involvement changing behaviour in practice or in improving outcomes for service users. Studies which have sought to evaluate the impact on practice (Rush, 2008; Turnbull and Weeley, 2013; MacSporran, 2015), are based largely on current students and the immediate impact. A number of researchers and authors therefore continue to identify the need to develop evidence for the efficacy of different types of involvement (Simons et al. 2006; Robinson and Webber, 2013) and what impact different types of involvement has on student learning and their subsequent practice in the long term (Muir and Laxton, 2012; Rhodes, 2012; Terry, 2012; Turnbull and Wheeley, 2013; Driessens, McLaughlin and Van Doorn, 2016).

This paper reports on a study which followed up a group of social work students to explore whether service user involvement in their education made any difference to their practice and whether there were different models or types of involvement which had a more significant impact on their practice than others.

\section{Terminology}


There is debate within the literature and in practice regarding the use of terminology for people who are the recipients of health and social work services or have a particular life experience from which students can learn. McLaughlin (2007) provides a useful critique of the most common terms and of how these represent the changing relationships within social work policy and practice (from client to consumer to service user to expert). In this paper the terms 'service user' and 'experts by experience' are both used due to their common usage within UK policy, practice and literature and the preferences of our partnership group. It is acknowledged however that these terms are not without their problems. As McLaughlin (2007, p.1108) suggests, the term service users 'neglects and denies the multiple socially constructed identities we all inhabit' whilst the term experts by experience fails to 'differentiate between the nature and types of experience'. Any term however that seeks to group together a diverse range of people and experiences will remain problematic. Attempts are made throughout this paper to provide more specific details so as not to generalise or to homogenise such a diverse group and to acknowledge the range of expertise and contributions individuals have made.

\section{Context}

The X University partnership group contributes to health and social work programmes across the Faculty of Health and Social Sciences and consists of over 90 active members (people who identify themselves as service users, carers or both). This is in addition to the contact students have with service users and carers whilst on placement; the involvement of service user led organisations who contribute directly to teaching and assessment on social work programmes and the knowledge students draw on from their own life experiences. Our model includes service users and carers sharing experiences, expertise and insight to enable students and academics to think critically and to reflect on their personal and professional 
development but also moves beyond this to include people as consultants; reviewers; researchers; facilitators and assessors due to the expertise they have and the perspectives they can share. We recognise that terms such as service user, patient, carer or experts by experience will include people who are also students; practitioners, academics and professionals; people of many skills and backgrounds and multiple roles and identities. We seek to involve a wide range of people in many different ways, to reflect this diversity and to address the need identified in the literature to involve seldom heard voices (Duffy, 2013) and to explore different models and strategies to avoid tokenism (Simons, Tee, Lathlean, Burgess, Herbert and Gibson, 2007). The types of involvement which students and graduates were asked to reflect on in this study therefore were diverse.

\section{Methodology}

The study adopted a narrative approach to seeking insight into the impact of service user involvement on a social workers' subsequent practice. A narrative approach seeks to gather a range of personal stories and accounts regarding a particular experience and explores these in order to gain understanding of a central issue (Creswell, 2002). By listening to participant stories, perspectives and critical reflections, we sought to increase our own insight and understanding but also facilitate the practitioners' own learning. Asking participants to share their own reflections, rather than to respond to a list of questions, placed more power with the individual participant and enabled them to identify what was significant for them. The open ended narrative inquiry approach led to contributions that as a researcher, I may not have thought to ask for such as the learning from service users whilst on placement or during post qualifying programmes such as the Approved Mental Health Profession (AMPH) training. 
The study invited final year social work students and qualified social workers to reflect on whether service user involvement in their education made any difference to their practice and whether there were different models or types of involvement which had a more significant impact on their practice than others. Participants were invited to submit a written narrative or attend a focus group or individual interview where they could be supported to share their stories and reflections. Requests to participate were distributed to final year social work students on the University's BA and MA Social Work programmes and to qualified social workers through our practice partners and the workforce development teams of four local authorities.

Ethics approval was gained through the University ethics procedure and involved providing potential participants with a participant information sheet which outlined the purpose and methods of the study; how their contributions would be used and of any disadvantages or advantages they might want to consider before choosing to take part. These included consideration of the impact of my dual role as researcher and senior lecturer on the qualifying programmes and my existing relationship with them. All participants were required to provide written consent for their contribution to be shared and for their anonymised narratives or excerpts from their narratives or contributions to be published.

The email requests resulted in the involvement of 20 participants; six students approaching the end of their qualifying programme; two newly qualified social workers undertaking their Assessed and Supported Year in Employment (ASYE); six social workers who had qualified within the last ten years (since the implementation of the degree and the requirement for involvement in social work education) and six social workers who had qualified prior to 2005 on DipSW or CQSW programmes. 17 of the participants were on placement or working within local authority teams (eight in children's services and ten in adults); two were working 
as freelance Practice Educators and independent social workers and one for an independent fostering agency. 13 of the participants were students or graduates of X University where I am practice lead and seven had graduated from other English Universities. 16 of the participants contributed to focus groups (two held at the university and one at the central offices of a local authority). One participant was interviewed individually at her workplace and three final year students contributed written narratives.

Verbal narratives were recorded and transcribed and a thematic analysis (Braun and Clarke, 2006, 2015; Fugard and Potts, 2015) was then applied to the written narratives and transcriptions to identify key themes and patterns. Propositional statements were then generated from the research findings to foster further analysis and reflection. Propositional statements are suggestions which draw on ideas and theories generated by the knowledge gained so far (Heron, 1996; Author's own, 2012) and then shared with others for further consideration. As others apply their own meaning, further knowledge and insight can be generated. The propositional statements created from this study form the conclusion of this paper.

\section{Findings and discussion}

Of the 20 participants, all identified significant learning from the involvement of service users on their programme with the exception of three who recalled minimal involvement other than at the admissions stage. All 20 participants contributed examples of learning from service users in practice settings. Learning had come from different types of involvement and with participants identifying a number of ways that their learning had impacted on their subsequent practice. These were subsequently categorised under four key themes: 
- Enhanced awareness of the lived experience

- Taking on board suggestions of good practice from service users and carers

- Developing a more critical 'real life' understanding

- A culture of recognising service users and carers as experts

\section{Enhanced awareness of the lived experience}

Within the data previously collected from students at the point of activity on my own programmes, the key area of learning for students was an enhanced awareness of the lived experience. This was still evident for students and practitioners reflecting on the impact of this involvement on their subsequent practice. One participant reflected on how some of the personal experiences shared in lectures had been very difficult to listen to. She remembered one person:

"she was in hospital for years and years and years and finally she' $\mathrm{d}$ been allowed out and it was like her whole life had been taken up by this institution, and I think just having someone to really delve deeper into that kind of experience - this is where I was, this is how I was feeling, this is what social services were telling me but this is actually the reality. She felt trapped and that no-one was listening to her. It was just really honest. Brutal stories can really help. I think for me they stick in my mind when I meet someone else I think oh yeah, I wonder if you're feeling like that right now".

One MA final year student reflected on how an encounter with a family foster carer whilst on placement enabled her to gain better insight: 
"She told me about how upset she was when she first started out as the training was the same for both the main stream foster carer and the family foster carers and how they were often referred to as 'cases' when they really were her grandson and family members that in reality were being talked about.

It really helped me to understand the difficulties that were unique to family foster carers as they were also dealing with the breakdown of relationships in the family, disappointment, anxiety and fear, as these were all her loved ones.

When I came to do my own Form F assessment on a family foster carer, I believe I was more prepared and could reflect this understanding of the carer's dilemmas when I provided an analysis in my assessment".

All of the participants who remembered involvement in lectures spoke positively about it. 'People coming in was a real highlight of our course' or 'we used to really look forward to those sessions'. One social worker commented on

"the courage it took for some of them to talk about something that was often a painful memory and quite personal to them but it helped me to gain insight into their life and also to think about what someone wants from their social worker".

One of the social workers explained how she considered it a privilege to hear about someone's lived experience.

\section{Taking on board suggestions of good practice from service users and carers}

Participants frequently referred to opportunities where they had been able to engage in discussions and consultations with service users and carers and ask questions about their 
experiences of social work and practitioner involvement. The significant factor in these examples was the level of interaction and engagement. A number of participants referred to specific sessions and people who had contributed, quoting them on suggestions or guidance they had offered. An ASYE now working in older people's mental health services said she had appreciated service users coming into lectures and talking honestly about what they thought of services. She referred to a mental health service user who had said she used to fear social services coming because of not being in control, not knowing what they were going to do and feeling ashamed about her continued self-harm.

"That for me helped my practice in the fact that I'm very mindful now that there are going to be people that don't want social services anywhere near them"

She explained how it made her more aware of how people react to the words 'social worker' or 'social services' and the need to "come at it from a different angle and build a rapport first". She explained how that single conversation had enabled her to manage expectations particularly around fear of social workers, particularly for older people in a mental health setting where she now works. She said

$$
\begin{aligned}
& \text { "some do genuinely think that they're going to end up in a straitjacket } \\
& \text { somewhere on lots and lots of pharmaceutical drugs and not seeing their } \\
& \text { families and that's quite scary for them". }
\end{aligned}
$$

She reflected on whether this lecture had felt so significant at the time: "I don't think it did because I didn't want to work in mental health at the time but it obviously did stick with me". A number of participants identified examples which had become more significant once they encountered the situation for themselves in their practice and engaged in a double loop of the learning cycle having been able to put their learning into practice (Kolb, 1984). In one of the 
narratives a final year student explained how in her first year she had conducted an assessed role play with someone who used substance use services.

"I did not know at the time, that I would end up in a drugs service, but my role play scenario was based around an addiction situation and as part of the feedback (the service user) talked to me a little about what it was like to work with people with addictions and I have since been able to reflect on the information he shared and how relevant it is to my current experiences working with addiction clients".

She goes on to explain that having the opportunity to learn from young people in her first placement setting with a range of experiences including Autism and mental health issues and the reading she then did to learn more about these experiences and what a diagnosis meant for different people, not only built her confidence but also "enabled me to be more empathetic in my current role".

A number of the participants identified aspects of their practice which had been informed by contributions on their course from service users and carers. A final year MA student said

"We had an afternoon where we had lots of different service users come in and carers from all sort of different areas of social services. Having a conversation with a carer and with a service user who both really highlighted that the worst thing a social worker can ever do is to say 'I'll do something' and not do it'.

The student reflected on how in one example, the worker had been off sick and there were understandable reasons but at the end of the day, what had been promised didn't get done.

"I think that by having that conversation with someone who had clearly been hurt a lot by lots of different team members you could see that actually that whole relationship could have been made better. Their whole care had been impacted by that 
and I think that for me, even if you do go off sick or something happens if you've made a promise you have to make sure that's going to be kept by the team".

An ASYE in the same focus group explained that as a result, she has learnt since not to make promises and to be much more open with service users by explaining when she hoped to have something done by and to phone them if there was a delay or to apologise when something doesn't get done.

'I've had a few crises in the last week, so I'm really sorry but your case didn't get the full attention it should and I'm sorry about that'. They do appreciate me being honest with them".

An ASYE in adult services remembered the same activity where a number of service users had come in to a lecture to share their experiences and suggestions. What she had remembered most was people's frustration at having to go through the same information again and again and then being very aware of the same frustrations being shared by service users in her subsequent practice.

"you could really see that, they were like "why are you asking us the same questions that she asked me'? And the parents are like 'you've already gone through my direct payment like ten times, it's not changed, why am I doing it all over again, and again and again”.

This reminded her of the discussion during her course and the need for openness and honesty. This was also identified as an example of learning in another focus group. An adult social worker said as a result she now always makes a point of summarising the case histories and explaining that she has done this to each service user so they don't feel they need to start from the beginning. 
A number of participants identified the need to be honest and transparent with service users as being a key aspect of learning from their involvement in their course. One recounted a service user saying to her "if you are not going to be able to offer me a service, just tell me so I can sort something else out".

An ASYE in adult services said she still remembered one service user who had facilitated a group she was in during her first year (four years earlier) who talked about professionalism. She remembered him saying

“' a lot of social workers are coming pretending to be my friends, and are there so casually, I have a lot of people who coming in jeans and leggings. I don't want them to be my friend I want my social worker to look like a social worker, so can you remember that', and I always remember that actually. I think you know depending on who I'm seeing I always make sure I have my badge and think about how I dress. I'll always remember that actually."

A number of the participants explained that having different views shared by service users helped them to reflect. Also on the topic of dress code, participants in one focus group said that they had received conflicting advice from service users on their course about whether to dress casually or smartly and how that had enabled them to realise that everyone is different. One student said how in practice she had learnt to adapt what she was wearing to different activities and people, as a result of conversations with service users:

"when I was working at a school and a social worker came in to assess a child and they were dressed in a suit and I sort of observed the social worker but from afar as I had the kids and I looked and the mother just said to me 'he can't even get down to play with him 'cos he's in a suit, how's he going to interact with her'.', 
In a different focus group, a social worker now working in a child health and disability team remembered a mother coming in to a lecture and saying that she has had social workers who have been to her house and not even acknowledged her daughter because of her level of communication needs. The social worker explained how this had significantly impacted on the student group at the time and led to a discussion about the need for basic communication. A children's social worker said she always remembers a woman coming in who had survived domestic abuse.

"She had a huge impact on our student group especially when she said to us, 'don't ask us why we don't leave'. Now I say it to students and newly qualified workers don't ask them that, they can't tell you'. That one statement was huge."

An experienced adult social worker said hearing people's views enabled her to think about what is important to the person which might seem small and be overlooked by others such as 'having Wheetos for breakfast or a spicy lunch because they find other food to be bland. "I put things like that in the care plan”.

Whilst much of the learning related to developing good practice and upholding social work standards and professional values, many of the participants felt that service user involvement had enabled this to take place. One more experienced social worker who recalled there being minimal involvement on her qualifying programme said that without this, her current focus on involvement in her practice had only developed much later. 'It's far better that we now introduce this right from the start'.

\section{Developing a more critical understanding}


A number of the participants reflected on how opportunities to discuss issues or hear about people's experiences had enabled them to adopt a more critical approach to what they were reading and learning and an understanding of complexity which informed how they practice now. As with the examples above, this criticality often arose from service users presenting varied experiences or providing conflicting advice.

A BA final year student said about a session in her first year:

"Service users from a day centre came to discuss their experiences. I found this highly beneficial, it enabled me to recognise that what is written in literature is slightly simplistic compared to reality. Although literature is fantastic, it does not have the same influence as a service user discussing their experiences, which almost makes the information we have learnt more real and applicable. I feel like I now question literature a lot more, and think how would this affect the service user? or what does this mean for the service user?"

One participant, a mental health social worker who qualified five years ago at a university where she identified few examples of involvement, identified the risk to less experienced students like herself, if different perspectives were not presented.

"I think at the time I used to take everything a service user said as universal. For a long time I thought this one woman's experience of post-natal depression was how it was for all women".

\section{A culture of recognising service users and carers as experts}

Having a range of involvement across the programme appeared to have successfully developed and promoted a culture for many of the participants in recognising service users as 
experts by experience and to value the opportunity to learn from them rather than 'do social work to them'. One ASYE said that she had taken this approach into her practice and now routinely explored a person's views on social care with them and sought to develop a relationship which was based on this level of mutual respect before focussing on the assessment.

A final year MA student said that in her placement in a substance use team, she had organised a drop-in session for service users to share their experiences of services. She reflected on how powerful it had been to listen to people's experiences and their ideas on treatment services. It made her reflect on the importance of seeking meaningful feedback and engaging in conversations with people who are experts by experience.

"Before I was doing the course and I was working we used to give out feedback forms and it felt very hollow, it was kind of this thing that you said 'please bring this in and then send it back to the department' but when I did it used to feel like it was a way of criticising me if that makes sense, it didn't feel like useful feedback it was like a weird oh what if they say something but now feedback I get much more of the whole picture; the importance of it".

She explained that this had taken time. Students on my own programme are asked to identify a plan in each placement for how they are going to learn from the expertise of service users and then to review this at the end. When reflecting back on her first placement she said:

"I don't think I'd been in the placement long enough to really understand what I could learn from the people in the placement but I think this time round, because I already knew what things service users could potentially teach me I was more aware of the ways I could get taught those things by them." 
Two of the more experienced participants who had qualified over ten years ago, recognised this change of culture and said

"you see the difference in students now. They come in with an attitude toward service users of "what can I learn from you'."

An experienced social worker who subsequently set up a new service said that involvement had been fundamental to her learning in her DipSW and that it just became the way she worked. "It's absolutely fundamental, it underpins everything I do. Everything's in partnership". She remembered recording interviews with children whilst on placement and how this had enabled her to focus on the voice of the child. She remembered having to write a narrative of the child's voice and explained how she still does that in her practice today.

Another participant identified experiences which had challenged concepts of 'them and us' such as one social worker who referred to a post qualifying session when they were participating in a lecture on mental health and it was only at the end that the lecturer disclosed their own mental health history. "It breaks down stereotypes".

Individual impact

Whilst there were some key themes in terms of what impact involvement had, the findings showed that the impact was individual to each student or practitioner. It was often specific encounters or 'light bulb moments' which led to increased awareness or insight into aspects of their own practice particularly when encountering similar situations or when pressures of practice led to them losing sight of service user perspectives. This was particularly evident in the reflections around professionalism and 'making promises' or not doing what you had said you would do because of a heavy case load or crisis. 
"You try to do as much as you can because you don't want to let them down but in reality if a crisis comes in and you've had to drop everything you might have promised you'll call at three o'clock but you just have to accept it wasn't possible for you to do. You have to be honest and apologise".

A significant aspect of the findings were how even some years on, students and practitioners were able to identify specific encounters and conversations they remembered with service users and carers contributing to their course and how something they had said or what they themselves had learnt, had "stayed with them". "I can picture them now" was a phrase used by a number of the participants. The memories were often in relation to advice on how to improve their own practice which they have since taken on board.

\section{Learning from practice}

Many of the participants including those who had qualified over ten years ago remembered individual service users they had encountered on their practice placements and receiving feedback from them. Two of the social workers who qualified more than ten year ago identified how much more important involvement had become in recent years and how much more meaningful it has become in their own practice.

"You are just stagnating if you are not constantly looking to your service users to develop the service. It's got to be meaningful; what's working, what's not working, can we do things differently? I've always found it quite inspiring and motivating to be sitting alongside a service user as an equal. It's about their lives so until you can get alongside them and understand their perspective, how can you improve?"

A number of participants identified how much of their learning about service user perspectives had come from placement. One talked about being encouraged to immerse 
herself in deaf culture; another about how the nature of the 24 hour family centre in which he was placed enabled him to gain a huge amount of insight into people's lives and to learn from them. "The most meaningful conversations were at 2am in the morning when they had just been on the phone crying to their mum". When reflecting on the impact on his subsequent practice he said "I learnt to listen". Concern was expressed in this focus group as to whether opportunities to get alongside service users exist in current practice. Participants now working in children's services talked about the value of driving children to appointments. One said 'I've driven round three times on my way somewhere because of how much they were opening up". Impromptu opportunities to have conversations with service users were valued by many of the participants over programme requirements to obtain feedback. Three of the participants questioned the value of having to obtain written feedback from service users whilst on practice which had been a requirement on their programme at the time. Those who were able to devise evaluation forms with service users or engage in other strategies such as interviews and conversations found this to be much more useful. Across all of the focus groups and narratives, the opportunity to engage with service users was identified as the most meaningful.

\section{Limited impact}

Particularly evident from the narratives were the types of involvement that were not mentioned or were perceived by the participants as being less significant in their learning or the shaping of their subsequent practice. A significant area of service user involvement across social work programmes and a requirement in England as stipulated by the Health and Care Professions Council (HCPC) is the involvement of service users and carers in the recruitment process. On my own programme, levels of involvement at the admissions stage are extensive as the carer and service user partnership group lead and assess a group activity which 
involves interviewees viewing a short film or documentary excerpt relevant to social work practice and discussing the service user perspectives within it. The carer and service user group then facilitate and provide feedback on a written activity where applicants are required to write a first person narrative from the perspective of one of the people within the film. In addition to assessing written skills, the aim of the activity is to evaluate the applicants' use of empathy. Marks and feedback from both of these activities and an interview with an academic and a social work practitioner, are then reviewed by the service user and carer coordinators and the admissions tutor to finalise offer decisions.

Despite this level of involvement, the only participants who referred to the interview day within their narratives, interview or focus groups were ones where the service users had been involved in the interview panel rather than a group or written activity. The responses to this involvement varied:

"My first experience of service user involvement on the course was my initial interview. This had two responses, initially I felt glad as the service user had experience of mental health services and this was the area I was discussing. At the time, I felt having service user involvement enabled me to express my interest in the particular field, to the point where I forgot I was in an interview. I think this benefited me personally, as it broke down the barriers of nervousness and I become more relaxed. Although, following the discussion I was apprehensive that I had not focused on the academic perspective enough".

Whilst another participant (a social worker who qualified five years previously) who remembered a service user being on the interview panel said 
"They were just like another member of staff as they all took turns in asking questions. I suppose it did help when they asked me about my personal experience as I felt I could talk about that".

Another participant remembered there being a service user on the interview panel but wasn't sure if it had any impact on her directly "I don't remember coming out thinking specifically oh yeah it was really good to have that service user in for that".

On my own programme we have a number of activities where service users and carers assess students' work, which includes being on marking panels for assessed presentations; taking part and providing feedback on assessed role plays and in providing feedback on how effectively the service user voice is evidenced within students' practice portfolios. Of these activities, only the assessed role play which involves a high level of interaction and personal disclosure by the service user was recalled and explored in any depth by the participants. This was rated far more highly by current students and recent graduates who conducted assessed role plays with service users who had planned the activity and devised their own scenarios, than those who had conducted role plays within the student group with students taking on the service user role. Again, this was due to the activity being perceived as realistic, providing insight into the person's lived experience and students valuing the opportunity to discuss their 'practice' with the service user after it had ended. A social worker from a different university who had undertaken an assessed role play with service users questioned its value for those involved as she didn't feel there was this level of involvement or engagement and was left feeling concerned about the impact of multiple role plays on the individual service users taking part; a concern also reported by Duffy, Das and Davidson (2013) when reflecting on a similar activity. 


\section{Conclusions and Implications for educators}

The most common types of service user and carer involvement mentioned by participants were lectures and workshops where individuals shared their lived experience and their views on best practice; when gaining individual feedback on their assessed role play; during a practice learning in the community unit where groups are facilitated by a service user; and when engaging in consultations of what makes a good social worker as part of their practice learning. All of these examples involved direct contact and provided opportunities for students to gain insight and engage in communicable learning where learners seek to understand (Mezirow, 2003).

Opportunities to engage in conversations with people who they considered experts by experience are well supported by a number of adult learning theories. Knowles (1990) argued that for adults, learning needs to be purposeful, to draw on previous experiences, to recognise their readiness to learn and their orientation to learning (what is relevant to their life) and to recognise what motivates them to learn. For most of the participants in this study, engaging with service users enabled them to see the relevance of what they had been learning elsewhere but also to learn directly from them and to use it to inform their own views of the world and their developing practice. It enabled them to gain insight and make sense of their wider experiences. In many cases it enabled students to develop as empathic practitioners but also provided guidance on how to demonstrate this in their practice; how as well as why.

When exploring the lived experience, a number of the participants identified personal accounts which they had found 'shocking' or 'difficult to hear'. As one of the participants said however, 'brutal stories can really help'. This is supported by transformative learning theory where Mezirow (2003) places particular emphasis on perspective change and the transformation of beliefs, values and behaviour. Mezirow identified ten phases of the 
transformative learning process which he argued occur as an adult seeks to clarify meaning, starting with 'a disorientating dilemma, self-examination of feelings and a critical assessment of assumptions' (Mezirow, 2003 p.4). Whilst others have argued that it requires a less linear process (Cranton, 2002; Merriam and Ntseane, 2008; Merriam and Kim, 2008; Walton, 2010), these elements were present for many of the participants reflecting on their learning from service users sharing these insights.

The lack of evidence within the narratives of lasting impact from involvement in admissions, assessment of students work and practice and curriculum design raises a number of questions. Further exploration is required to identify whether this reflects students and practitioners' perceptions of involvement only being when a person shares their story and lived experiences, rather than their expertise and judgment; whether the role of service users is well enough communicated during admissions or assessment processes; or whether the benefit of these types of involvement is in how it informs the decision making process rather than the applicants' experience on the day or memory of it after. One of the service user and carer coordinators reflected on whether the high level of responsibility the service users have during admission and assessment processes, was in some ways 'too professional' in that the service users were perceived as staff and that role differed from what an applicant's expectations may be of a 'service user'. Anka and Taylor's (2016) review of service user involvement in social work assessments however, identified the presence of different power relations amongst those involved, with some students acknowledging that whilst they valued service user feedback, in summative processes, they perceived the academic or practice assessor's assessment as 'carrying more weight' (2016 p.179). This could explain the limited recollection or impact identified by participants within this study. 
A number of conclusions can be drawn from the research regarding the impact of service user and carer involvement on students' subsequent practice and the types of involvement that appeared to have more impact. In addition to the four themes identified from the participant stories, the following propositional statements were identified from the knowledge gained (Heron, 1996; Author's own, 2012). These are presented here by way of a conclusion to this paper and as recommendations for educators to foster further reflection and debate.

- The impact of different types of service user involvement on subsequent practice is unique to each student

- Opportunities to hear personal experiences and the lived experience can have a long lasting impact on students and 'stays with them'

- The most significant impact on subsequent practice often comes from service users advising students on best practice and what they want from a social work practitioner.

- Impact on subsequent practice is increased if the student experiences double loop learning (Kolb 1984) for example, when going on to encounter a similar experience or scenario in their practice

- Impact on subsequent practice is increased when students have the opportunity to engage and discuss issues with the service user. The impact comes from meaningful conversations

- Having a diverse range of involvement and the opportunity to hear contrasting views and perspectives can be an effective catalyst for critical reflection

- The reality of people's lives and their experiences of services and practitioner involvement can enable students to develop a less simplistic view of social work practice and a better understanding of complexity

- For some students, it is only later when in practice that they recognise the significance of particular encounters and their learning from involvement 
- Opportunities for meaningful conversations with service users to support a student's learning and professional development can be facilitated in practice and university settings.

\section{References}

Author's own (2012): [name deleted to maintain the integrity of the review process].

Anka, A and Taylor, I (2016) Assessment as the site of power: A Bourdieusian Interrogation of Service User and Carer Involvement in the Assessments of Social Work Students. Social Work Education: The International Journal, 35:2, 172-185

Braun, V and Clarke, V., (2006) Using thematic analysis in psychology, Qualitative Research in Psychology, 3:2, 77-101.

Braun, V. and Clarke, V. (2013) Successful qualitative research: A practical guide for beginners. London:Sage

Carr, S (2004) Has Service User Participation Made a Difference to Social Care Services? London, SCIE, Policy Press.

Cranton, P., (2002) Teaching for transformation. New Directions for Adult and Continuing Education, 93, 63-72.

Creswell, J. W., (2002) Research Design: Qualitative, Quantitative and Mixed Methods Design. London: Sage 
Driessens, K, McLaughlin, H and Van Doorn, L (2016) The Meaningful Involvement of Service Users in Social Work Education: Examples from Belgium and The Netherlands. Social Work Education: The International Journal, 35:7, 739-751

Duffy, J. (2013) Service user involvement in teaching about conflict - an exploration of the issues. International Social Work 55(5) 720-739

Duffy, J, Das, C and Davidson, G (2013) Service User and Carer Involvement in Role-plays to Assess Readiness for Practice. Social Work Education: The International Journal, 32:1, $39-54$

Fugard, A.J.B., and Potts, H.W.W., (2015) Supporting thinking on sample sizes for thematic analyses: a quantitative tool, International Journal of Social Research Methodology, 18:6, 669-684,

Heron, J., (1996) Co-operative Inquiry: Research into the Human Condition. London: Sage

Khoo, R., McVicar, A., Brandon, D., (2004) Service user involvement in postgraduate mental health education. Does it benefit practice? Journal of Mental Health, October 2004; 13(5): 481-492.

Knowles, M., (1990) The adult learner: A neglected species. $4^{\text {th }}$ ed. London: Gulf. Kolb, D. (1984) Experiential learning: Experience as the source of learning and development. New Jersey: Prentice Hall.

MacSporran, J (2015) A Mentor's PATH: Evaluating how service users can be involved as mentors for social work students on observational practice placements. Social work and Social Sciences Review, 17 46-60 
McLaughlin, H (2009) What's in a Name: Client, Patient, Customer, Consumer, Expert by Experience, Service User - What's Next? British Journal of Social Work, 39, 1101-1117

Merriam, S.B. and Kim, Y. S., (2008) Non-Western perspectives in learning and knowing. New Directions for Adult and Continuing Education, 119,71-81.

Merriam, S.B. and Ntseane, G., (2008) Transformative learning in Botswana: How culture shapes the process. Adult Education Quarterly, 58 (3), 183-197.

Mezirow, J., (2000) Learning as transformation: Critical perspectives on a theory in progress. San Francisco: Jossey Bass.

Mezirow, J., (2003) Epistemology of transformative learning. Available at: www.transformativelearning.org/.../Mezirow_EpistemologyTLC.pdf

Morgan, A and Jones, D (2009) Perceptions of Service User and Carer Involvement in Healthcare Education and Impact of Students' Knowledge and Practice: A literature review. Medical Teacher 31, 82-95

Muir, D and Laxton, C.J (2012) Experts By experience: the views of service user educators providing feedback on medical students' work based assessments. Nurse Education today, $32,146-150$

Perry, J., Gilbert, A., Rawlinson, J., (2013) A systematic review of the evidence on service user involvement in interpersonal skills training of mental health students. Journal of Psychiatic and Mental Health Nursing, 2013, 20, 525-540.

Rhodes, C.A (2012) User Involvement in Health and Social Care Education: A concept analysis. Nurse Education Today 32, 185-189 
Robinson, K and Webber, M (2013) Models and Effectiveness of Service User and Carer Involvement in Social Work Education: A Literature Review. British Journal of Social Work $43,925-944$

Rush, B., (2008) Mental health service user involvement in nurse education: A catalyst for transformative learning. Journal of Mental health, 17 (5), 531-542.

Simons, L., Tee, S., Lathlean, J., Burgess, A., Herbert, L., Gibson, C., (2007) A socially inclusive approach to user participation in higher education. Journal of Advanced Nursing 58 (3), 246-255

Terry, J (2012) Service User Involvement in Pre-registration Mental Health Nurse Education Classroom Settings: a review of the literature. Journal of Psychiatric and Mental Health Nursing, $19816-829$

Turnbull, P and Weeley, F.M (2013) Service User Involvement: Inspiring student nurses to make a difference to patient care. Nurse Education Practice 13 454-458

Walton, J.D., (2010) Examining a transformative approach to communication education: A teacher-research study. College Student Journal, 44 (1), 157-177. 